\title{
Optimization of a Train Traffic Management Problem under Uncertainties and Disruptions
}

\author{
Adnen EL AMRAOUI ${ }^{1}$, Khaled MESGHOUNI ${ }^{2}$ \\ 1 Université d'Orléans, Laboratoire PRISME, \\ 63 Avenue de Lattre de Tassigny, 18020 Bourges, France, \\ adnen.el-amraoui@univ-orleans.fr \\ 2 Ecole Centrale de Lille (EC-Lille), Cité Scientifique - CS 20048, \\ 59651 Villeneuve d'Ascq Cedex, France, \\ khaled.mesghouni@ec-lille.fr
}

\begin{abstract}
In this paper, we consider a train traffic management problem. Our aim is to find an optimal schedule for a train traffic network where time duration uncertainties are considered. This problem was intensively studied with mixed integer linear models where trains moving duration are deterministic. In this paper, a new formulation of the problem as a classical one with scenario-based stochastic programming taking expected values as objective functions is presented. Then, new criterion is proposed to quantify scheduling robustness in the face of uncertainty. Besides, a novel control policy is elaborated to find quickly, a feasible train schedule when disruption or unexpected event occurs during scheduling execution.
\end{abstract}

Keywords: Train Traffic Management, Scheduling, Control Strategy, Uncertainties, Disruptions.

\section{Introduction}

This paper deals with the train scheduling networks problems. It consists in finding the arrival and the departure times of the lines at certain stages of the network. Depending on required objectives, these stages can be referred to public station and/or switches.

Since 1871 s, and more precisely since the first train schedule conference in Germany, train scheduling problems have been widely studied [1] and several mathematical models have been proposed ([2]-[3]-[4]-[5]-[6]-[7]).

This category of scheduling problems can be shared into two classes:

\section{$1^{\text {st }}$ Class: Static or Predictive problem}

It consists firstly on allocating resources (i.e. tracks and stations) to all trains in all routes. Then, the train sequencing entrains the prespecification of the arrival and departure order of trains at stations. Finally, a time-table is then resulted. This class aims on the minimization of the makespan or the cycle time or on the maximization of the traffic frequency [8]-[9][10]. In [9], Harrod propose a directed hypergraph formulation for a rail network, in the aim to schedule train paths when the railway network is busy.

Harrod model is used to solve the problem of train sequencing constraints. Moreover, a heuristic approach is then derived for the same problem [9].
Besides, a heuristic approach is proposed by Kraay and Harker to find line dispatching (i.e. arrival and departure times for each train) and to define a monthly strategic schedule [10].

Nevertheless, these approaches are not able to solve the problem when an expected event happens.

\section{$2^{\text {nd }}$ Class: Dynamic or Reactive problem}

It involves when the train planned schedule cannot be respected due to a disturbance handling activity. In this case, a new timetable should be found while all the problem constraints are respected. Generally, the objective function consists on the minimization of train delays [11]-[12]-[13].

Dorfman and Medanic propose a discrete event model to solve their strategy (called feedbackbased travel advance strategy). Moreover, they suggest some extensions of their strategy for more complex configurations (e.g. double-track sections, trains with variable characteristics and priorities) [11]. Narayanaswami and Rangaraj develop a mixed integer linear programming model to find a solution based on their strategy of controlling disjunctive constraints (of tracks allocation) [12]. Whereas, Budai et al. [13] use a timetable planning schedule as an input and apply a control strategy to minimize the delay. In this control strategy, trains movement sequence order is not challenged.

The focus of our paper is to present a new model which can be useful for the two problem classes 
simultaneously. Furthermore, the originality's of our approach consist on the following:

- Due to train travelling duration's uncertainties, a handling scenarios approach is presented. Moreover, additional criterion is considered to find the most robust schedule.

- In order to remove a disruption, if any, a new control policy is proposed. This policy aims to control train speed and train waiting time on stations.

Most previous models are handling either predictive train scheduling problem ([6]-[7][8]-[9]-[10]) or reactive one ([11]-[12]-[13]).

If unexpected event happens, a first schedule solution can be determined using the first problem class models. This can be reached by instantiating known decision variables. Nevertheless, this solution is very simple and cannot, at any way, guarantee the solution performance.

Besides, weather conditions can require on trains to reduce their speeds on some tracks. In fact, a wheels sliding or a wheels skating can happen due to the snow or to the tree leaves on the rail in autumn generally. So, this could lead to several perturbations in arrival and departure times of the timetabling passenger trains. This problem has become recurrent in Europe at the approach of winter holidays and Christmas while a large number of people take the train to travel; which make rail transport less competitive compared to other means of travel (air and ground transportation).

Following to these introductory remarks, Section 2 is devoted to the problem statement. Section 3 discusses the mathematical problem modelling. The problem resolving methodology and the new control policy are presented in Section 4 and 5, respectively.

\section{Problem Description}

In this study, we consider the single track, bidirectional railway traffic. Trains have to travel in two directions: from right to left $(R t o L)$ and from left to right (LtoR) (called also nominal direction [9]). Each left to right direction train is travelling, as soon as possible from the starting station (station 1), then it is visiting successively $m-1$ stations, numbered from 1 to $m-1$, before arriving to the end station (station $m$ ). While each right to left direction train has to start by the end station, and then it is visiting the stations: $m-1, m-2 \ldots$ and 2 successively, before reaching the starting station. We call single track (or segment) the slice of the line confined between any two stations. In general, on each train station several tracks (called block) are available to allow overtakes and crossings.

One of the main specificities of such system is that the average train travelling durations values are known and any delay can make a network disruption. Moreover, tracks are the most critical resource of such lines. Besides, there are no multiple-tracks between stations and each station can receive more than one train at the same time.

Figure 1 shows an example of a line layout with single track and bi-directional train movements.

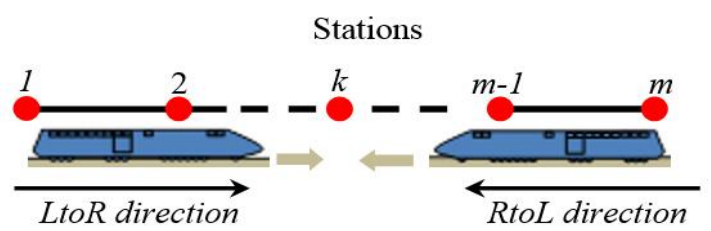

Figure 1. Example of a single track, bidirectional railway.

This problem can be considered as a job-shop scheduling problem with very specific constraints, where each segment is considered as a machine and each train as a job.

The constraints we consider here are the following ones:

- (C1) Each track can receive simultaneously either RtoL trains or LtoR trains.

- (C2) In each station, trains must remain at least a lower duration and at most an upper duration. These durations can vary from one station to another due to station passengers' frequency.

- (C3) Between two successive trains moving on the same direction, a minimum safety time duration is required.

- (C4) In each meeting station, minimum meeting time duration has to be ensured between the arrival and the departure of trains moving in different direction. Definitely, passages have to be allowed to change from one train to another.

The studied problem requires two distinct but dependent decisions to be made: (1) scheduling decision-sequence, in which trains have to move (priority to move), and (2) station waiting decision (real waiting time on stations). The strong dependence between these two decisions 
makes the problem very hard to model and then to solve. Yet, getting motivated by previous researches using mixed integer linear programming methods, we elaborate a new model; which can solve this train scheduling problem to optimality. In this programming model, we are looking for optimizing the simultaneous travelling durations of several trains moving in different directions through a single line. Besides, it can be also useful to find a solution quickly, when a disturbance occurs.

In general, the problem solution is presented using graphic timetable as shown in Figure 2.

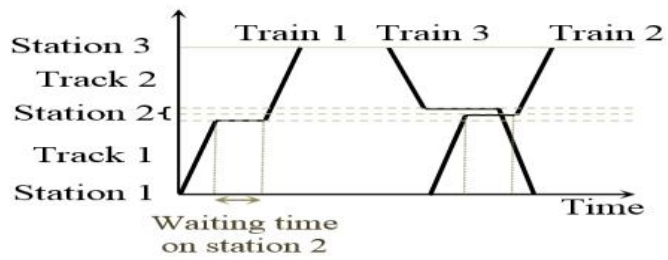

Figure 2. Graphic timetable.

This figure illustrates an example of a single track line layout with three stations and three trains (two moving from left to right: Train 1 and Train 2; and one from right to left: Train 3 ). Slash lines represent the train moves, while horizontal lines show the waiting times on stations. In this example, only one train meeting is carried out on station 2 between train 3 and train 2. After the passage of the train 1 , train 3 reaches station 2, wait there until the track 1 becomes available and the minimum meeting time takes in. Then, it leaves station 2 to station 1. While train 2 spends the lower bound of its required waiting time on station 2 and go to station 3 .

In the following section, we propose to describe the problem and formulate it as a mixed integer linear programming model.

\section{Problem Modelling}

A definition of notations used in this paper is necessary in order to describe the elaborated model. So, let's define the following parameters and variables:

\subsection{Parameters}

1. $n$ - Total number of trains.

2. $n_{1}$ - Trains that move in the LtoR direction.

3. $n_{2}$ - Trains that move in the RtoL direction.

4. $m$ - Total number of stations.
NOTE 1:

- $\quad$ Stations are indexed from 1 to $m$.

- To simplify the following notation, we denote by $\vec{i}$ a train moving in LtoR direction and by $i$ a train moving in RtoL direction.

5. $\quad U p t d_{i}^{k}$ The upper and lower bounds ${ }_{L} t^{k}$ travelling time of a $L t o R$ train

$L w t d_{i}^{k} i$ to run through the track between the stations $k$ and $k+1$.

6. $\quad U p t d_{i}^{k}$ The upper and lower bounds Lwtd $^{k}$ travelling time of a $R$ toL train $i$ to run through the track between the stations $k$ and $k$ 1 .

7. $U p w t_{i}^{k}$ The upper and lower bounds Lwwt ${ }^{k}$ waiting time of LtoR train $i$ on station $k$.

8. $\quad U p w t_{i}^{k}$ The upper and lower bounds $L w w t^{k} \quad$ waiting duration of RtoL train

9. $\quad \operatorname{Sfte}_{i, j}^{k}$ The safety time durations between the arrival of two

$S f t e_{i, j}^{k} \quad$ trains $(i$ and $j$ ) of the same direction to station $k$ :

$$
\begin{aligned}
& S f t e_{i, j}^{k}=\left|e_{i}^{k}-e_{j}^{k}\right| \\
& S f t e_{i, j}^{k}=\left|e_{i}^{k}-e_{j}^{k}\right|
\end{aligned}
$$

10. $S f t s_{i, j}^{k}$ The safety time durations between the departure of two $S f t s_{i, j}^{k} \quad$ trains $(i$ and $j)$ of the same direction from station $k$ :

$$
\begin{aligned}
& S f t s_{i, j}^{k}=\left|s_{i}^{k}-s_{\vec{j}}^{k}\right| \\
& S f t s_{i, j}^{k}=\left|s_{i}^{k}-s_{\dot{j}}^{k}\right|
\end{aligned}
$$

11. $\operatorname{Mmt}_{i, j}^{k}$ The minimum durations for ${ }^{i, j} t^{k}$ the meeting of two trains ( $i$ $M m t_{i, j}^{k}$ and $j$ ), moving on the same direction, on station $k$.

12. $M m t_{i, j}^{k}$ The minimum durations for $M m t^{k}$ the meeting of two trains $(i$ and $j$ ), moving on different directions, on station $k$.

13. $M \quad$ Very big number $(+\infty)$.

14. $D d_{i}^{k}$ Date when a disturbance occurs in the network for train $i$

$D d_{\tilde{i}}^{k} \quad$ on or after visiting station $k-1$.

15. $\delta_{i}^{k}, \delta_{i}^{k} \quad$ Disturbance duration. 


\subsection{Decision variables}

16. $\operatorname{Rtd}_{i}^{k}$

Real travelling time of a $L t o R$

$\operatorname{Rtd}_{i}^{k}$ and a $R$ to $L$ train $i$ from station $k$ to the following one.

17. $R w t_{i}^{k} \quad$ Real waiting time of a $L t o R$ and $R w t_{i}^{k} \quad$ a RtoL train $i$ on station $k$.

18. $s_{i}^{k}$

Start moving time of a train $i$ from station $k$ to station $k+1$.

19. $s_{i}^{k} \quad$ Start moving time of a train i from station $k$ to station $k-1$.

20. $e_{i}^{k} \quad$ End moving time of a train $i$ from station $k$ to station $k+1$.

21. $e_{i}^{k}$

End moving time of a train $i$ from station $k$ to station $k-1$.

22. $T g s_{i}^{k} \quad$ Term gain speed from station $k$ $\operatorname{Tgs}_{i}^{k}$

23. $T g w_{i}^{k} \quad$ Term gain waiting on station $k$.

$\operatorname{Tg} w_{i}^{k}$

24. $S_{\vec{i}, j}^{k}$

$$
= \begin{cases}1 & \text { if }\left(s_{i}^{k}<s_{\vec{j}}^{k}\right) \\ 0 & \text { otherwise }\end{cases}
$$

25. $E_{i, j}^{k}$

$$
= \begin{cases}1 & \text { if }\left(e_{i}^{k}<e_{\vec{j}}^{k}\right) \\ 0 & \text { otherwise }\end{cases}
$$

26. $S_{i, j}^{k}$

$$
= \begin{cases}1 & \text { if }\left(s_{i}^{k}<s_{j}^{k}\right) \\ 0 & \text { otherwise }\end{cases}
$$

27. $E_{i, j}^{k}$

$$
= \begin{cases}1 & \text { if }\left(e_{i}^{k}<e_{\grave{j}}^{k}\right) \\ 0 & \text { otherwise }\end{cases}
$$

28. $S_{\vec{i}, j}^{k}= \begin{cases}1 & \text { if }\left(s_{i}^{k}<s_{\dot{j}}^{k}\right) \\ 0 & \text { otherwise }\end{cases}$

29. $E_{i, j}^{k} \quad= \begin{cases}1 & \text { if }\left(e_{i}^{k}<e_{i j}^{k}\right) \\ 0 & \text { otherwise }\end{cases}$

\section{NOTE 2:}

- We assume that trains travel on a single track line layout in bi-directional movements. In addition, all trains have to pass through all stations. Moreover, re-routing is not allowed and safeties as well as lower time durations have to be respected.

- It is important to mention that the following mathematical formulation is a train movement's model: where decision variables define the starting dates of all the train moves from each station $k$ and their end dates $\left(s_{i}^{k}, s_{j}^{k}, e_{i}^{k+1}\right.$ and $\left.e_{j}^{k-1}\right)$.

\subsection{Formulation}

Minimize: $C_{\max }=\sum_{i=1}^{n_{1}} e_{i}^{m}+\sum_{j=1}^{n_{2}} e_{j}^{l}$

$\forall k: 1 \rightarrow m, \vec{i}: 1 \rightarrow n_{1}$ and $\overleftarrow{j}: 1 \rightarrow n_{2}$

$s_{i}^{k-1}+R t d_{i}^{k}=e_{i}^{k}$

$s_{\grave{j}}^{k+1}+\operatorname{Rtd}_{\grave{j}}^{k}=e_{\dot{j}}^{k}$

$\operatorname{Lwtd}_{i}^{k} \leq \operatorname{Rtd} d_{i}^{k} \leq U p t d_{i}^{k}$

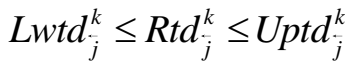

$e_{i}^{k}+R w t_{i}^{k} \leq s_{i}^{k}$

$L w w t_{i}^{k} \leq R w t_{i}^{k} \leq U p w t_{i}^{k}$

$L w w t_{\stackrel{j}{j}}^{k} \leq R w t_{\stackrel{j}{k}}^{k} \leq U p w t_{\grave{j}}^{k}$

$s_{\vec{j}}^{k}+S f t s_{i, j}^{k} \leq s_{i}^{k}+S_{i, j}^{k} \cdot M$

$s_{\vec{i}}^{k}+S f t s_{i, j}^{k} \leq s_{\vec{j}}^{k}+\left(1-S_{\vec{i}, \vec{j}}^{k}\right) \cdot M$

$e_{\vec{j}}^{k}+S f t e_{i, j}^{k} \leq e_{i}^{k}+E_{i, j}^{k} \cdot M$

$e_{i}^{k}+S f t e_{i, j}^{k} \leq e_{j}^{k}+\left(1-E_{i, j}^{k}\right) \cdot M$

$s_{\stackrel{j}{k}}^{k}+S f t s_{i, j}^{k} \leq s_{i}^{k}+S_{i, j}^{k} \cdot M$

$s_{\grave{i}}^{k}+S f t s_{i, j}^{k} \leq s_{\grave{j}}^{k}+\left(1-S_{i, j}^{k}\right) \cdot M$

$e_{i j}^{k}+S f t e_{i, j}^{k} \leq e_{i}^{k-1}+E_{i, j}^{k} \cdot M$

$e_{i}^{k}+S f t e_{i, j}^{k} \leq e_{\dot{j}}^{k-1}+\left(1-E_{i, j}^{k}\right) \cdot M$

$s_{\vec{j}}^{k}+M m t_{i, j}^{k} \leq e_{i}^{k}+S_{i, j}^{k} \cdot M$

$e_{i}^{k}+M m t_{i, j}^{k} \leq s_{j}^{k}+\left(1-S_{i, j}^{k}\right) \cdot M$

$e_{\stackrel{j}{k}}^{k}+M m t_{i, j}^{k} \leq s_{i}^{k}+E_{i, j}^{k} \cdot M$

$s_{i}^{k}+M m t_{i, j}^{k} \leq e_{j}^{k}+\left(1-E_{i, j}^{k}\right) \cdot M$

$S_{i, j}^{k}=E_{i, j}^{k}$

$S_{i, j}^{k}=E_{i, j}^{k}$

$S_{i, j}^{k}=E_{i, j}^{k}$ 


$$
\begin{aligned}
& e_{i}^{m}=s_{\vec{i}}^{m} \\
& s_{\leftarrow}^{1}=e_{j}^{1} \\
& S_{\vec{i}, j}^{k} ; E_{i, j}^{k} ; S_{\vec{i}, j}^{k} ; E_{i, j}^{k} ; S_{\vec{i}, \dot{j}}^{k} ; E_{\vec{i}, \bar{j}}^{k} \in\{0,1\}
\end{aligned}
$$

In this model, the objective function (1) consists in minimizing the makespan $C_{\max }$. This variable, as given here, can be defined as the total time needed for all the trains to reach their terminals. Constraints (2)-(3) guarantee the fact that: before arriving to the destination station each train has to spend its required travelling time (i.e. the most frequent duration). This duration must be confined within lower and upper bounds as traduced by constraints (4)-(5). Constraint (6) defines the stations waiting dates that must be respected. These durations have to be bounded, as defined by constraints (7)-(8). Before starting to move, each train must be sure that the minimum safety time duration between him and the previous one on the same track is maintained. This statement is translated by constraints (9)-(12) for LtoR trains and by constraints (13)-(16) for RtoL trains. While constraints (17)-(20) are used to ensure the minimum required time duration at a station between the arrival and the departure of two trains moving in different directions. Constraints (21)-(23) define the precedence rule: if a train $i$ leaves first a station $k$, it must reach first the destination station (i.e. before train $i+1)$. Consistency constraints are given by (24) and (25), while binary decision variables are defined by (26).

NOTE 3: Unlike all previous mixed integer linear programming models dealing with train scheduling problem [3]-[5]-[6]-[10]-[11]-[12], this optimizing model highlights several flexibilities of the considered problem. Eqs. (4), (5), (7) and (8) are called Time Windows (TW) constraints and traduce these flexibilities.

\subsection{Model resolving}

To solve this Mixed Integer Linear Programming (MILP) model, a commercial software solver, Cplex of IBM, is used and a branch-and-bound procedure, implemented on it, is applied to explore the train schedules.

This procedure is elaborated here with a simplified arbitration strategy which considers disjunctions according to the track plane of figure 1 and which gives priority to the train first starting move. The illustration of the search tree is presented in Figure 3.

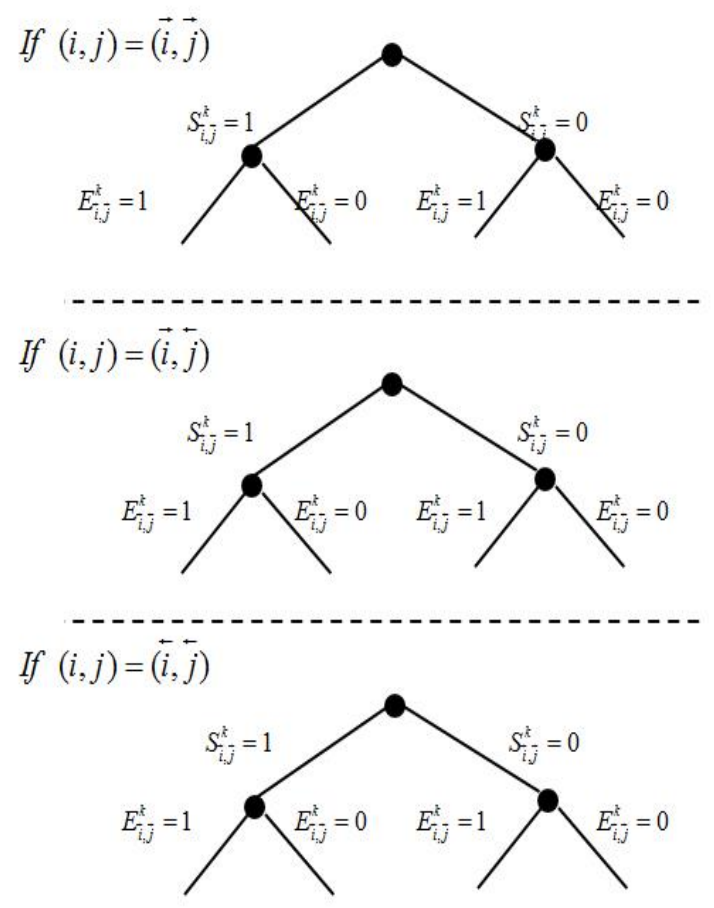

Figure 3. Illustration of the constraint propagation mechanism.

Besides, in order to limit the number of nodes, the evaluation phase is supposed to use a constraint propagation mechanism. Thereby, for each train station $\mathrm{k}$, two nodes are considered. Each one corresponds to the arbitration of one of the disjunctions (i.e. order of arrival and departure for each pair of train $(i, j))$. That is to say, disjunctive constraints constitute the branches of the tree, while the potential schedules are defined by the tree leafs.

Then, for each leaf of the search tree and when all the disjunctive constraints are considered, the binary variables are instantiated and the MILP model is launched (Eqs. (1)-(26)). If it has a solution, the considered schedule is feasible and unfeasible otherwise.

Finally, the best feasible solution is returned as the optimal solution of the problem.

\subsection{Complexity}

The complexity of this problem is related to the number of trains, directions and stations (or / and tracks).

For this proposed scheduling model, there are $m\left(27 n_{1} n_{2}+4 n_{1}+4 n_{2}\right)+n_{1}+n_{2}$ constraints. 
Besides, there are four types of decisions variables: a total of $4 m\left(n_{1}+n_{2}\right)+1$ integer variables and $6 m n_{1} n_{2}$ binary variables to find.

\subsection{Formulation limits}

This model can be used in general to find a timetabling for a single track line layout, with bi-directional train movements problem. Nevertheless, due to some unexpected events, train traffic can be disturbed. Consequently, a new, quick and robust schedule solution should be found.

For this aim, we propose: firstly, a new solving methodology for the problem on hand, using metrics that takes into account the scheduling characteristics under train transportation time uncertainty and we illustrate it by an example. Secondly, an evolution of the previous model is performed to make it able to find efficient schedule solution, when an unforeseen event happens. This aim can be achieved by applying a new control policy based on: speed train control and train waiting time control. These studies will be presented consecutively, in the following sections.

\section{Problem Resolving Methodology}

Getting motivated by such transportation scheduling problems and previous robustness studies in optimization literature and more precisely in job-shop scheduling problems [13][14], a scenario based stochastic problems methodology is proposed in this section to define the fastest and the most robust schedule.

The performance of the schedule can be evaluated in terms of the makespan required while random transportation durations are satisfied. In this proposed methodology, train travelling durations are assumed to vary by $P \%$ about their nominal values. Realistically, this probability may be calculated for each line track and each period of the year, on the basis of the historic of railways transportation companies (i.e. SNCF in France). Then, for each one of the $S$ considered case studies, the MILP model is launched to find the optimal solution $\left(C_{\max , s}\right)$ which has a probability of $p_{s}$ to take place. Afterward and when all these scenarios are simulated, the sought schedule can be found by applying the procedure of Algorithm 1.

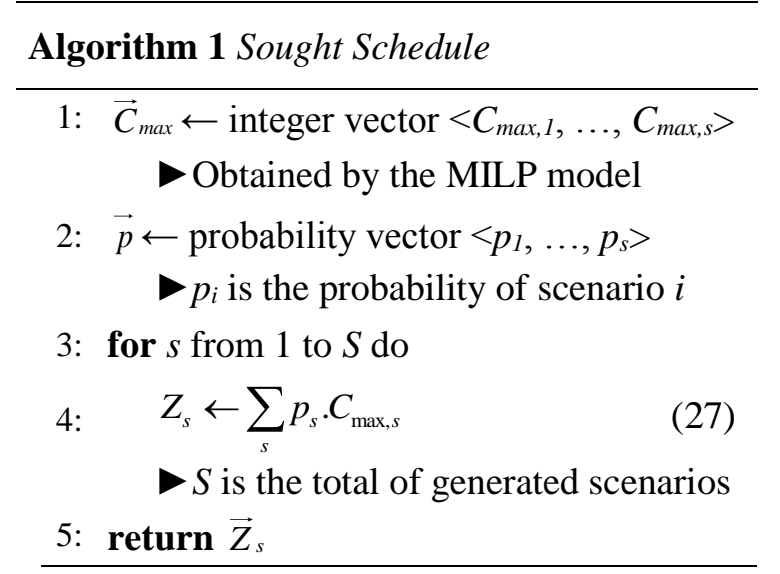

Furthermore, the deal consists on finding the most robust schedule. This robustness, as defined for same problems, measures the resilience of the schedule objective to vary under uncertain parameters and disruptive events. Moreover, as explained previously, the weather can have a big impact on the train time travelling durations and consequently on the disturbance network. Thus, in order to determine the more resilient schedule, the standard deviation is proposed here as a metric for robustness evaluation.

Therefore, the most robust schedule can be found using the procedure of Algorithm 2.

Algorithm 2 Robust Schedule

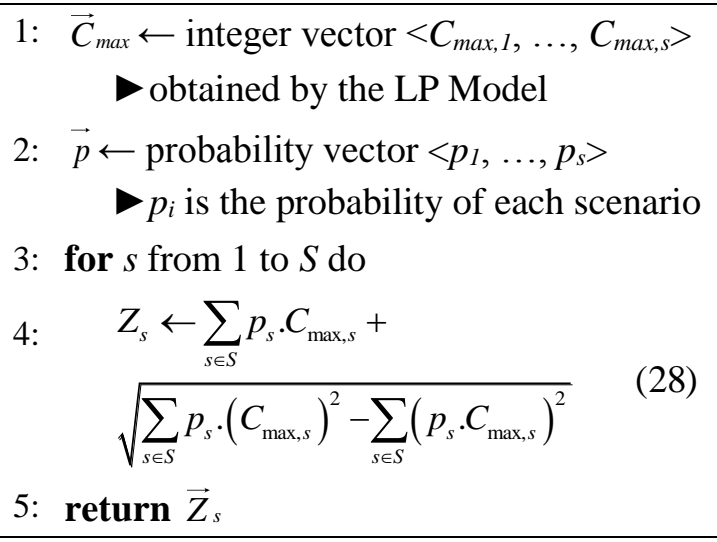

The proposed resolution methodology is very helpful for the decision maker to find the best schedule. In other word, it can help him to find the most probably solution with the fastest completion time, where time durations uncertainties are considered. Moreover, the solution stability is also evaluated using a new metric (see Eq. (28)) in order to help the decision maker to choose the more flexible schedule (e.g. which can be affected less than the others by any disturbance). 
To illustrate this new resolution methodology, an illustrative example is proposed in the following subsection.

\subsection{Illustrative example}

In this example three trains have to travel on a line with 4 stations including the starting and the ending stations. Train 1 and 2 travel on LtoR direction, while train 3 travels on RtoL direction. The travelling time durations are assumed to vary from $0 \%$ to $25 \%$ about their nominal values. These distributions are given in Table 1.

Table 1. Travelling time distribution

\begin{tabular}{|c|c|c|l|}
\hline Train & Track & $\begin{array}{c}\text { Nom. } \\
\text { Value }\end{array}$ & $\begin{array}{c}\text { Real } \\
\text { Value }\end{array}$ \\
\hline \multirow{3}{*}{$\overrightarrow{1}$} & 1 & 45 & Rand( $\left.\mathrm{N}_{\mathrm{V}} ; 1.25 \mathrm{~N}_{\mathrm{V}}\right)$ \\
\cline { 2 - 4 } & 2 & 28 & Rand $\left(\mathrm{N}_{\mathrm{V}} ; 1.25 \mathrm{~N}_{\mathrm{V}}\right)$ \\
\cline { 2 - 4 } & 3 & 50 & Rand $\left(\mathrm{N}_{\mathrm{V}} ; 1.25 \mathrm{~N}_{\mathrm{V}}\right)$ \\
\hline \multirow{3}{*}{$\overrightarrow{2}$} & 1 & 47 & Rand( $\left.\mathrm{N}_{\mathrm{V}} ; 1.25 \mathrm{~N}_{\mathrm{V}}\right)$ \\
\cline { 2 - 4 } & 2 & 27 & $\operatorname{Rand}\left(\mathrm{N}_{\mathrm{V}} ; 1.25 \mathrm{~N}_{\mathrm{V}}\right)$ \\
\cline { 2 - 4 } & 3 & 49 & $\operatorname{Rand}\left(\mathrm{N}_{\mathrm{V}} ; 1.25 \mathrm{~N}_{\mathrm{V}}\right)$ \\
\hline \multirow{3}{*}{$\overline{3}$} & 1 & 45 & Rand $\left(\mathrm{N}_{\mathrm{V}} ; 1.25 \mathrm{~N}_{\mathrm{V}}\right)$ \\
\cline { 2 - 4 } & 2 & 26 & $\operatorname{Rand}\left(\mathrm{N}_{\mathrm{V}} ; 1.25 \mathrm{~N}_{\mathrm{V}}\right)$ \\
\cline { 2 - 4 } & 3 & 40 & $\operatorname{Rand}\left(\mathrm{N}_{\mathrm{V}} ; 1.25 \mathrm{~N}_{\mathrm{V}}\right)$ \\
\hline
\end{tabular}

NV: Nominal Value (Nom. Value)

Rand (): Randomly generated value.

The parameter Probability in this table could define the frequency of spending these time durations over a period of time. This probability can vary from one season to another due to weather conditions for example. The total number of considered scenarios is 96 . And the remaining problem parameters used for simulations are given as follows:

$$
L w w t_{i}^{k}=5 ; S f t e_{i, j}^{k}=1 ; S f t s_{i, j}^{k}=2 ; M m t_{i, j}^{k}=3 \text {. }
$$

Applying the proposed MILP model and the Algorithm 1 procedure to these data example, several makespans are obtained. These makespans values are varying from 428 to 453 t.u. with various probabilities of occurrence, as reported in Figure 4.

According to this histogram, it is easy to notice that the most expected values of the makespan is 445 t.u. which is defined in literature as the deterministic value of the makespan [13]. In other words, it determines the most likely schedule to be followed, where probabilistic travelling time durations are considered.

Then, in the aim to quantify robustness, the standard deviation metric is used in this example by applying the procedure of the
Algorithm 2. The simulation results of Figure 5 show that the most robust schedule is for the makespan of 446 t.u.

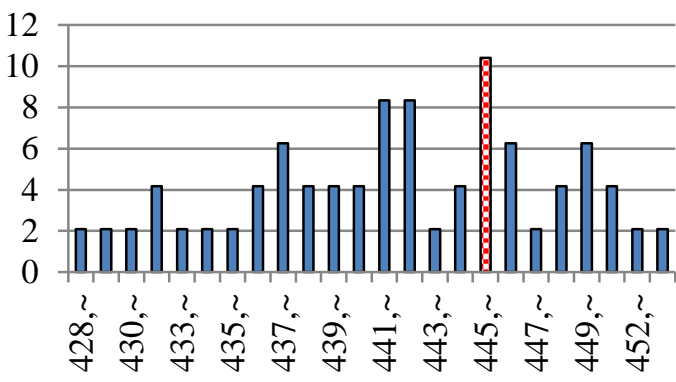

Figure 4. Probability of scenarios expected makespans.

It is clear that under these conditions, the most frequent and fastest schedule is corresponding to the makespan of 445 t.u. Nevertheless, it is not the more robust one (see Figure 5).

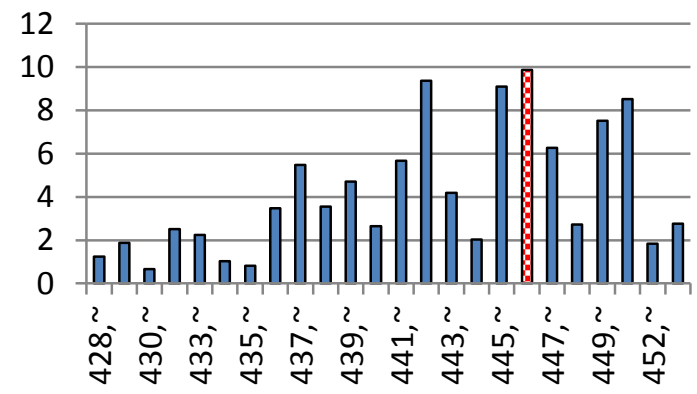

Figure 5. Probability of scenarios expected makespans plus standard deviation.

In this case study, the decision maker has to choose between these schedules: the most frequent schedule with a fast completion time and the most robust one. Nevertheless, this choice is not always easy to do, as it seems in this example. Hence, a tradeoff between these two criteria (fast completion and robustness and/or reliability) has to be held.

Graphic timetable of the makespans 445 t.u. and 446 t.u. are reported on Figure 6-(a) and 6(b), respectively.

Moreover, we use direct graph models to characterize the difference between slight and heavy robustness (see figure 6-(c)).

In this figure, we model priority moving constraints; and as we can see, schedule 1 presents more precedence constraints than schedule 2 . In fact, in schedule 2, train 1 leaves station 3 before the arrival of train 2. Moreover, the safety time duration for the starting move of train 2 is achieved before it arrives to the departure station which makes it free to move at any time. Thus, schedule 
1 is a slight robust schedule whereas schedule 2 is a heavy robust one.

After making his choice, the decision maker has to apply the selected train timetable schedule. Despite of the high level of solution robustness, disruptions can happen and can affect very badly the train traffic network. For this aim, a control policy for railway traffic management is proposed in the following section.

\section{Control Policy}

Such as several previous approaches [3]-[4][6]-[8]-[9]-[10], the MILP model of sections 3 is incapable to face disruptive events when there is a perturbation on the train network. Thus, to make it able to find new feasible and performing schedules, if conflicts occur due to one or several delays, several new constraints are added to solve these inconsistencies.

Two alternatives of control policy can be applied to find quickly a good solution:

\section{1st alternative of control policy}

It consists on the propagation of the delay on all the over train travelling dates. For example, if a disturbance $\delta_{i}^{k}$ happens at $D d$ for a train $i$.

All the transportation movements scheduled before $D d$ are not altered. Despite the others, they have to be delayed by $\delta_{i}^{k}$.

This solution can be applied but the optimality is not guaranteed. Moreover, the propagation of the delay can have a snowball effect. Therefore, the train delays and the refund fees of railway companies will increase drastically.

\section{$2^{\text {nd }}$ alternative of control policy}

It consists on updating all the starting move dates of all the trains. To apply this policy, a train speed control and a train waiting time control strategies are applied. These strategies can be traduced in our MILP model by the following constraints:

$\overline{s_{i}^{k-1}}+R t d_{i}^{k}+\delta_{i}^{k}-T g s_{i}^{k}=\overline{e_{i}^{k}}$

$\operatorname{Lwtd}_{i}^{k} \leq \operatorname{Tg} s_{i}^{k}$

$\operatorname{Tgs}_{i}^{k} \leq U p t d_{i}^{k}$

$\overline{s_{j}^{k+1}}+R t d_{j}^{k}-T g s_{\dot{j}}^{k}=\overline{e_{j}^{k}}$

$\operatorname{Lwt} d_{\stackrel{j}{j}}^{k} \leq \operatorname{Tgs}_{\breve{j}}^{k}$

$\operatorname{Tgs}_{j}^{k} \leq U p t d_{j}^{k}$

$\overline{e_{i}^{k}}+R w t_{i}^{k}-T g w_{i}^{k} \leq \overline{s_{i}^{k}}$

$T g w_{i}^{k} \leq U p w t_{i}^{k}$

$L w w t_{i}^{k} \leq T g w_{i}^{k}$

NOTE 4: We denote by $\bar{x}$ the new decision variable value of $x$ when a regulation is performed after a disturbance. In other words $\bar{x}$ is the new value of $x$ after a new schedule.

Constraints (29), (32) and (35) have to be used instead of constraints (2), (3) and (6). And new time windows constraints ((30), (31), (33), (34), (36) and (37)) have to be added.

Let's detail and analysis these new constraints. We assume that a disruption happens when a $L$ toR train $i$ leaves a station $k-1$, then the arrival date of this train at station $k$ will be equal to: $\overline{s_{i}^{k-1}}+R t d_{i}^{k}+\delta_{i}^{k}=\overline{e_{i}^{k}}$ in spite of equality (2).

Moreover, due to ground topology and thanks to train technology evolution, a first control strategy can be applied. In fact, each train can increase its speed on some segments of its course. Generally, this speed is not deterministic but confined on a speed interval: limited by lower and upper bounds. So, using

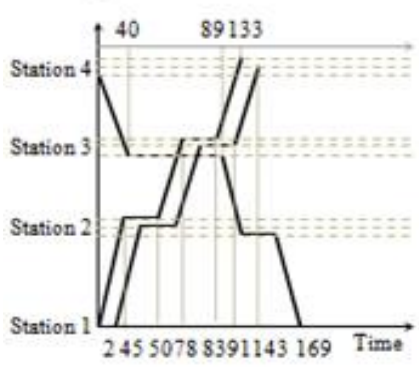

(a)

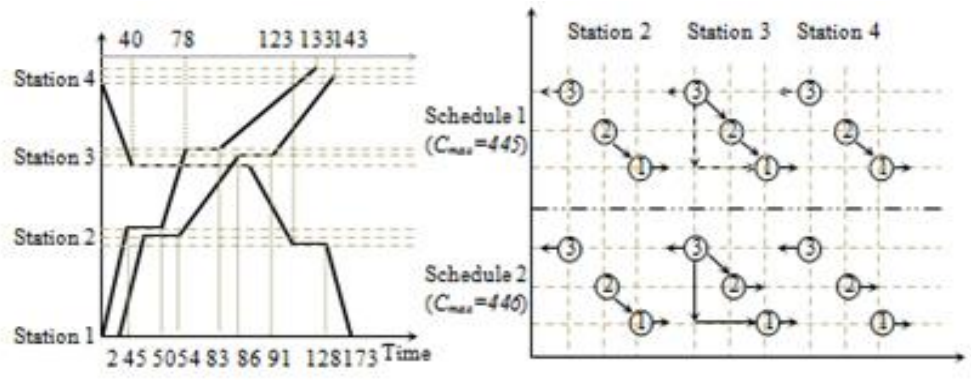

(b) (c)

Figure 6. Graphic timetable and robustness analysis 
this problem specificity, we introduce a new parameter called term gain speed $\left(T g s_{i}^{k}\right)$ to determine the gain that it can be reached when the train speeds are well controlled. Consequently, equalities (29) and (32) have to be used instead of constraints (2) and (3).

Besides, train speed limits have to be respected. For this aim, we introduce the new constraints (30) and (33).

The second control strategy proposed in our MILP model is the waiting time control technique. In fact, for this class of scheduling problem, a waiting time is defined on each train station. This time duration can be decreased, to absorb the time disruption, provided that a minimum waiting duration on each station is respected. These statements are traduced by constraints (35), (36) and (37).

To illustrate the effectiveness of the control policy, the following example is proposed and several disruption cases are presented.

\subsection{Illustrative example}

Similarly to the illustrative example of section 3 , let's consider the same track configuration (with 4 stations) and let's consider that two trains are moving in LtoR direction while a third one is moving on RtoL direction.

The travelling time durations are assumed to be equal to the nominal value, as given by table 1 . Further, the following data: time windows and parameters values; have to be defined according to the defined control strategy.

- Time windows values:

$$
\begin{aligned}
& 10 \leq \operatorname{Rtd}_{i}^{k} \leq 15 ; 5 \leq R w t_{i}^{k} \leq 15 ; 0 \leq \operatorname{Tgs}_{i}^{k} \leq 2 ; \\
& 15 \leq \operatorname{Rtd}_{j}^{k} \leq 20 ; 5 \leq R w t_{j}^{k} \leq 15 ; 0 \leq \operatorname{Tg} s_{j}^{k} \leq 2 . \\
& 0 \leq \operatorname{Tg}_{i}^{k} \leq 3 ; 0 \leq \operatorname{Tg} w_{j}^{k} \leq 3 .
\end{aligned}
$$

- Parameters values:

$$
\begin{aligned}
& S f t e_{i, j}^{k}=1 ; S f t s_{i, j}^{k}=2 ; M m t_{i, j}^{k}=3 ; \\
& S f t t_{i, j}^{k}=1 ; S f t s_{i, j}^{k}=2 ; M m t_{i, j}^{k}=3 .
\end{aligned}
$$

Let's analyze the following cases study:

\subsection{Case 1. No disturbance.}

Using the elaborated linear programming model, while disruption and gains are omitted, the optimal solution is obtained for a $C_{\max }$ of 147 t.u. as reported on figure 7-(a).

\subsection{Case 2. A disturbance of 5 t.u. for train 3 at time $\mathrm{Dd}=10$.}

Thanks to the speed and waiting time control strategies, the optimal solution remains the same (see figure 7-(b)).

In fact, thanks to the defined train gain speed time window $\left(0 \leq T g s_{j}^{k} \leq 2\right)$, we can win 2 t.u. and then, we reduce the delay to 3 t.u. only. Moreover, the train gain waiting window $\left(0 \leq T g w_{j}^{k} \leq 3\right)$ allows us to absorb the 3 t.u. remaining of the delay.

\subsection{Case 3. Moreover the departure of train 2 is delayed to 25 .}

As illustrated on figure 7-(c), by applying our control strategy we find a schedule where only the train 3 (train where disturbance has occurred) was delayed.

However, it is very important to notice that we gain about $2,3 \%$ on the $C_{\max }$, which cannot be neglected, compared to the solution where control strategies are not applied.

In conclusion, on these cases study, we illustrate by a scholar example the effectiveness of the proposed control policy to find a performing schedule solution when an expected event happens.

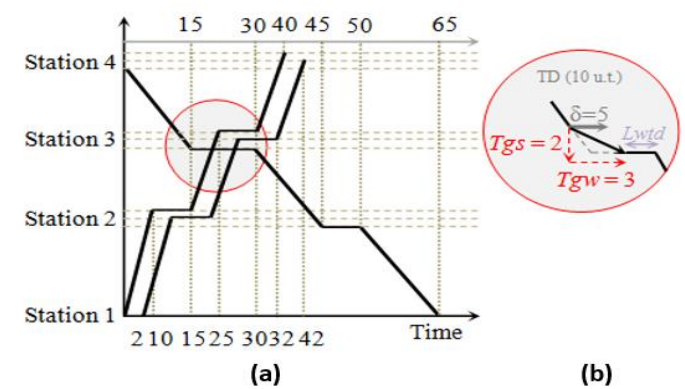

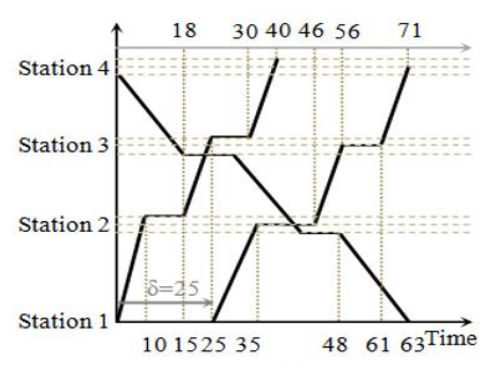

(c)

Figure 7. Graphic timetable when the control policy is applied. 


\section{Conclusion}

In the aim to optimize a train traffic scheduling problem under time travelling uncertainty, we proposed a scenario based stochastic problems methodology to solve the problem. Moreover we defined new objective function criteria to quantify the schedule robustness. Besides, we extended the proposed Mixed Integer Linear Programming model to solve the problem on hand where unexpected event happens. For this aim, we developed a control policy based on train speed and waiting time control strategies.

The proposed control policy shows its efficiency on terms of solution quality (e.g. total delay and number of delayed trains).

For future work, we propose to extend the elaborated control policy model, to more complex lines' configurations (e.g. doubletrack sections, trains with variable characteristics and priorities).

\section{Acknowledgements}

This research was supported in part by the engineering school "Ecole Centrale de Lille (EC-Lille)" under a grant from the ANR Project, named (PERFECT).

\section{REFERENCES}

1. CORDEAU, J. F., P. TOTH, D. VIGO, A Survey of Optimization Models for Train Routing and Scheduling, Transp. Sc., vol. 32, 1998, pp. 380-404.

2. BAI, L., T. BOURDEAUD'HUY, B. RABENASOLO, E. CASTELAIN, A Mixed-integer Linear Program for Routing and Scheduling Trains Through a Railway STATION, 3rd Intl. Conf. on Operations Research and Enterprise Systems, Angers, 2014, pp. 445-452.

3. HIGGINS, A., E. KOZAN, L. FERREIRA, Optimal Scheduling of Trains on a Single Line Track. Transp. Res. - Part B, vol. 30(2), 1996, pp. 147-161.

4. SHERALI, H. D., R. SIVANANDAN, A. G. HOBEIKA, A Linear Programming Approach for Synthesizing OriginDestination Trip Tables from Link Traffic Volumes, Transportation Research B, vol. 28, 1994, pp. 213-233.
5. CHANG, S., Y.-C. CHUNG, From Timetabling to Train Regulation- A New Train Operation Model, Information and Software Technology, vol. 47(9), 2005, pp. 575-585.

6. ASSAD, A. A., Models for Rail Transportation. Transportation Research Part A, vol. 14, 1980, pp. 205-220.

7. NACHTIGALL, K., S. VOGET, Minimizing Waiting Times in Integrated Fixed Interval Timetables by upgrading Railways Tracks, European J. of Oper. Research, vol. 103, 1997, pp. 610-627.

8. HARROD, S., Modeling Network Transition Constraints with Hypergraphs, Transportation Science, vol. 45, no. 1, 2011, pp. 81-79.

9. ABBAS-TURKI, A., O. GRUNDER, A. EL MOUDNI, Sequence Optimization for Timetabling High Frequency Passenger Trains, IEEE Joint Rail Conf., Philadelphia, Penn., 2012, pp.831-836.

10. KRAAY, D. R, P. T. HARKER, RealTime Scheduling of Freight Railroads. Transportation Research - Part B, vol. 29, no. 3, 1995, pp. 213-229.

11. DORFMAN, M. J., J. MEDANIC, Scheduling Trains on a Railway Network using a Discrete Event Model of Railway Traffic, Transportation Research - Part B, vol. 38, 2004, pp. 81-98.

12. NARAYANASWAMI, S., N. RANGARAJ, Modelling Disruptions and Resolving Conflicts Optimally in a Railway Schedule. Computers \& Industrial Engineering, vol. 64, 2013, pp. 469-481.

13. BUDAI, G., G. MAROTI, R. DEKKER, D. HUISMAN, 1. KROON, Rescheduling in Passenger Railways: The Rolling Stock Rebalancing Problem, Journal of scheduling, 2009, pp. 281-297.

14. VIN, J. P., G. IERAPETRITOU, Robust Short-Term Scheduling of Mutiproduct Batch Plants under Demand Uncertainty, Ind. Eng. Chem. Res., vol. 40, 2001, pp. 4543-4554.

15. HARDING, S. T., C. A. FLOUDAS, Global Optimization in Multiproduct and Multipurpose Batch Design under Uncertainty, Ind. Eng. Chem. Res., 36, 1997, pp. 1644-1664. 